\title{
GERENCIANDO A EQUIPE DE ENFERMAGEM NA SALA DE EMERGÊNCIA
}

\author{
Managing the nursing staff in the emergency room
}

Eliszangela Daniel Monsani ${ }^{1}$, Maria Tereza Soratto²

* Artigo Baseado na Monografia de Pós-graduação Especialização em Urgência e Emergência.

${ }^{1}$ Enfermeira. Pós Graduada em Assistência de Enfermagem em Urgência e Emergência.- Universidade do Extremo Sul de Santa Catarina - UNESC- Criciúma SC - Brasil.Email: edmonsani@gmail.com

${ }^{2}$ Enfermeira. Mestre em Educação - UNESC - Universidade do Extremo Sul de Santa Catarina - Criciúma - SC - Brasil. Email: guiga@unesc.net.

\section{Endereço para correspondência:}

Maria Tereza Soratto. Departamento de Enfermagem - UNESC. Av. Universitária, 1105. Criciúma - SC - Bairro Universitário. CEP - 88806-000. Email: guiga@unesc.net. 


\section{Resumo}

Este estudo objetivou complementar o conhecimento do enfermeiro enquanto gerenciador da equipe de enfermagem na sala de emergência. Trata-se de uma pesquisa de revisão integrativa, de caráter descritivo, qualitativo, desenvolvido a partir de artigos científicos publicados entre 2013 à 2017, indexados na Scielo (Scientific Eletronic Libraly Online); e Google acadêmico a fim de se identificar publicações envolvendo a temática sobre o gerenciando da equipe de enfermagem na sala de emergência. Identificou-se 869 artigos, sendo analisados 10 artigos. 0 enfermeiro desenvolve um papel fundamental enquanto gerenciador da equipe de enfermagem no setor de urgência e emergência. Atividade esta que vem ser facilitada com a experiência profissional. $O$ enfermeiro gerenciador precisa ter habilidade na resolutividade de conflitos, capacidade técnica e ser para a equipe um líder participativo em todas as situações, resultando assim em um trabalho prazeroso para todos os envolvidos.

Palavras-chave: gerenciamento; enfermagem; urgência; emergência.

\section{Abstract}

This study aimed to complement the knowledge of nurses as nursing team manager in the emergency room. It is an integrative review research, descriptive, qualitative character, developed from scientific articles published between 2013 to 2017, indexed in Scielo (Scientific Electronic Online Libraly); and Google scholar to identify publications involving the theme about the managing of the nursing staff in the emergency room. 869 articles identified, being analyzed 10 articles. The nurse develops a key role as team manager of nursing in the urgency and emergency. This activity come be facilitated with professional experience. The nurse manager must have skills in conflict resolution, have technical ability and be a team leader participating in all situations, thus resulting in a pleasurable work for everyone involved.

Keywords: Management; Nursing; Emergencies. 


\section{INTRODUÇÃO}

Nos serviços hospitalares vivenciamos diariamente situações de urgência e emergência, a qual nos faz repensar as atividades técnicas, com o âmbito de melhorar a assistência prestada aos pacientes que procuram ou que são conduzidos aos serviços. Situações de urgência se caracterizam por uma ocorrência imprevista de um agravo à saúde do ser humano com ou sem risco potencial de vida, cujo portador desta situação necessita de assistência imediata. Enquanto que a emergência é uma condição onde se constata um risco de vida iminente e sofrimento intenso ${ }^{1}$. Neste setor é necessária a aplicação de práticas clínicas cuidadosas e ágeis. Para tanto, os setores de urgência e emergência são ambientes onde os profissionais devem ter a capacidade de tomar decisões rápidas, serem tecnicamente qualificados e trabalharem em harmonia, pois irão prestar cuidados de enfermagem de maior complexidade técnica e para pacientes graves.

Aplicar a Sistematização de Assistência de Enfermagem (SAE) em uma unidade de Urgência e Emergência não se caracteriza em uma atividade fácil, visto que a procura pelas unidades tem crescido gradativamente. Outro fator relevante dase ao fato de que surgem constantemente situações que nos desafiam, em uma gama de novas patologias e agravos. Manter a equipe de técnicos de enfermagem, bem como os demais membros da equipe multiprofissional, enquanto enfermeiro responsável tem sido um trabalho inovador. $O$ trabalho desses profissionais exige esforço físico, mental, emocional e psicológico, visto que demanda atenção, realização de atividades com alto grau de responsabilidade e dificuldade, ritmo acelerado de trabalho, jornadas excessivas e poucas horas de descanso.

Percebe-se, que como um profissional gerenciador de cuidados, 0 enfermeiro é um ator importante na assistência prestada, principalmente nos serviços de urgência e emergência. Levando-se em consideração que o enfermeiro é o único profissional de nível superior da equipe de enfermagem e, portanto, o responsável por ela. Sua atividade como um bom gerente será o foco de um bom trabalho².

A partir da experiência profissional como enfermeira atuante em unidade de emergência em um Hospital de pequeno porte, observou-se a necessidade de 
qualificar o gerenciamento da equipe de enfermagem no atendimento prestado aos pacientes em situações de urgência e emergência. Neste contexto tem-se como pergunta norteadora da pesquisa: como gerenciar a equipe de enfermagem na sala de emergência enquanto profissional enfermeiro?

Considera-se que o gerenciamento da equipe pode melhorar a qualificação da equipe técnica; resolutividade dos casos; agilidade nos atendimentos e encaminhamentos com maior segurança. Nesta perspectiva tem-se como objetivo realizar uma revisão de literatura sobre o gerenciando da equipe de enfermagem na sala de emergência.

\section{MATERIAIS E METODOS}

Trata-se de uma pesquisa de revisão integrativa, de caráter descritivo, qualitativo, desenvolvido a partir de artigos científicos publicados entre 2013 à 2017, indexados na Scielo (Scientific Eletronic Libraly Online); e Google acadêmico a fim de se identificar publicações envolvendo a temática sobre o gerenciando da equipe de enfermagem na sala de emergência.

A revisão integrativa é um método de pesquisa apontada como uma ferramenta que utilizada na busca, avaliação crítica e síntese das evidências disponíveis do tema investigado 3,4 .

A revisão integrativa é constituída pelas seguintes etapas: identificação do tema e problema de pesquisa, estabelecimento de critérios de inclusão e exclusão, definição das informações a serem extraídas dos artigos selecionados com a coleta de dados, análise das informações, interpretação dos resultados e apresentação da revisão ${ }^{3-6}$.

Para a localização dos artigos publicados na base de dados utilizaram-se os seguintes critérios de inclusão: artigos que relatam o assunto publicado no banco de dados da Scielo (Scientific Electronic Library Online); e Google acadêmico; descritores - palavras-chaves: gerenciamento; enfermagem; urgência; emergência; sala de emergência; língua portuguesa; artigos publicados no período de 2013 à 2017. 
Os critérios de exclusão foram relacionados aos artigos não disponibilizados na íntegra; artigos repetidos; resenhas; editoriais e dossiês; teses e dissertações; além daqueles que não se enquadravam no ano pesquisado.

O processo de coleta de dados ocorreu de acordo com a seguinte sistematização: a avaliação inicial do material bibliográfico mediante a leitura dos resumos, com a finalidade de selecionar aqueles que atendiam aos objetivos do estudo, através do tema proposto, onde foram totalizados 869 artigos. A seguir realizou-se a leitura dos artigos selecionados na íntegra, com a seleção final de 10 artigos para análise.

\section{RESULTADOS E DISCUSSÃO}

A apresentação da revisão e a discussão dos dados foram realizadas de forma descritiva, sendo analisados 10 artigos que atenderam os critérios de inclusão. Os artigos utilizados na revisão foram organizados e sintetizados em um quadro contendo informações de acordo com título, tipo e método do estudo, ano, autores, periódico.

Quadro1 - Artigos selecionados de acordo com título, tipo e método do estudo, ano, autores, periódico

\begin{tabular}{|c|c|c|c|c|}
\hline Título do artigo & $\begin{array}{c}\text { Tipo e método do } \\
\text { estudo }\end{array}$ & Ano & Autores & $\begin{array}{c}\text { Artigo } \\
\text { Periódico }\end{array}$ \\
\hline $\begin{array}{l}\text { A liderança do } \\
\text { enfermeiro no contexto } \\
\text { dos serviços de } \\
\text { urgência e emergência }\end{array}$ & Artigo de revisão & 2014 & ${\text { SILVA et } \text { al }^{1}}^{1}$ & $\begin{array}{l}\text { Rev. Eletr. } \\
\text { Enf. }\end{array}$ \\
\hline $\begin{array}{lr}\text { A qualidade } & \text { da } \\
\text { assistência } & \text { de } \\
\text { enfermagem em } & \text { uma } \\
\text { unidade } & \text { de } \\
\text { emergência: } & \text { a }\end{array}$ & $\begin{array}{l}\text { Exploratório } \\
\text { descritivo; quantitativo } \\
\text { com } 260 \text { usuários de } \\
\text { uma Unidade } \\
\text { Emergência Adulto }\end{array}$ & 2013 & $\begin{array}{l}\text { MORAES; } \\
\text { MELLEIRO7}^{7}\end{array}$ & $\begin{array}{l}\text { Rev. Eletr. } \\
\text { Enf. }\end{array}$ \\
\hline
\end{tabular}

Revista Inova Saúde, Criciúma, vol.9, n. 1, jul. 2019. 


\begin{tabular}{|c|c|c|c|c|}
\hline ário & & & & \\
\hline $\begin{array}{lr}\text { Acolhimento } & \text { e } \\
\text { classificação de risco } \\
\text { nos serviços de } \\
\text { urgência } \\
\text { emergência: limites e } \\
\text { possibilidades uma } \\
\text { questão para os } \\
\text { enfermeiros }\end{array}$ & $\begin{array}{l}\text { Descritivo exploratório e } \\
\text { qualitativo, aplicação de } \\
\text { questionários } \\
\text { observação não- } \\
\text { participante/ } \\
\text { assistemática, com } 11 \\
\text { enfermeiros }\end{array}$ & 2014 & $\begin{array}{l}\text { SANTOS et } \\
\mathrm{al}^{8}\end{array}$ & $\begin{array}{l}\text { Persp. On } \\
\text { line: biol. } \\
\text { Saúde }\end{array}$ \\
\hline $\begin{array}{l}\text { Estratégias utilizadas } \\
\text { pelos enfermeiros para } \\
\text { promover o trabalho } \\
\text { em equipe em um } \\
\text { serviço de emergência }\end{array}$ & $\begin{array}{l}\text { Pesquisa qualitativa do } \\
\text { tipo estudo de caso } \\
\text { realizada com } 20 \\
\text { enfermeiros do Serviço } \\
\text { de Emergência }\end{array}$ & 2016 & $\begin{array}{l}\text { SANTOS et } \\
\mathrm{al}^{9}\end{array}$ & $\begin{array}{l}\text { Rev. } \\
\text { Gaúcha } \\
\text { Enferm. }\end{array}$ \\
\hline $\begin{array}{l}\text { Exercício da liderança } \\
\text { do enfermeiro em um } \\
\text { serviço de urgência e } \\
\text { emergência }\end{array}$ & $\begin{array}{l}\text { Descritivo, exploratório, } \\
\text { qualitativo, aplicação de } \\
\text { entrevista com } 10 \\
\text { enfermeiros do serviço } \\
\text { de urgência } \\
\text { emergência }\end{array}$ & 2016 & $\begin{array}{l}\text { AMESTOY } \\
\text { et } \mathrm{al}^{10}\end{array}$ & $\begin{array}{l}\text { Rev. Elet. } \\
\text { Gestão e } \\
\text { Saúde }\end{array}$ \\
\hline $\begin{array}{l}\text { Gerenciamento de } \\
\text { enfermagem de } \\
\text { unidade } \\
\text { emergência: } \\
\text { dificuldades e desafios }\end{array}$ & $\begin{array}{l}\text { Estudo de caso: } \\
\text { descritivo e qualitativo, } \\
\text { aplicação de entrevista } \\
\text { semiestruturada com } 06 \\
\text { enfermeiros do setor de } \\
\text { emergência de um } \\
\text { hospital }\end{array}$ & 2013 & $\begin{array}{l}\text { ZAMBIAZI; } \\
\text { COSTA }^{11}\end{array}$ & חAS \\
\hline $\begin{array}{l}\text { Prazer e sofrimento no } \\
\text { exercício gerencial do } \\
\text { enfermeiro no contexto } \\
\text { hospitalar }\end{array}$ & $\begin{array}{l}\text { Exploratório descritivo, } \\
\text { qualitativo, aplicação de } \\
\text { entrevista } \\
\text { enfermeiros de um } 19\end{array}$ & 2013 & $\begin{array}{l}\text { SANTOS et } \\
\mathrm{al}^{12}\end{array}$ & $\begin{array}{l}\text { Esc. Anna } \\
\text { Nery }\end{array}$ \\
\hline
\end{tabular}




\begin{tabular}{|c|c|c|c|c|}
\hline & hospital universitário & & & \\
\hline $\begin{array}{l}\text { Relacionamento } \\
\text { interpessoal entre a } \\
\text { equipe de uma } \\
\text { emergência hospitalar: } \\
\text { um estudo qualitativo } \\
\text { sob o olhar de } \\
\text { enfermeiros }\end{array}$ & $\begin{array}{l}\text { Estudo qualitativo, } \\
\text { descritivo e exploratório, } \\
\text { com } 14 \text { enfermeiros }\end{array}$ & 2015 & $\begin{array}{l}\text { LIMA NETO } \\
\text { et } \mathrm{al}^{2}\end{array}$ & Rev. Enf. \\
\hline $\begin{array}{lr}\text { Sistematização } & \text { da } \\
\text { assistência } & \text { de } \\
\text { enfermagem e } & \text { o } \\
\text { gerenciamento } & \text { do } \\
\text { cuidado: revisão } & \text { de } \\
\text { literatura } & \end{array}$ & Artigo de revisão & 2016 & CARVALH $^{13}$ & $\begin{array}{l}\text { Rev. Elet. } \\
\text { SIMTEC }\end{array}$ \\
\hline $\begin{array}{lr}\text { Percepção } & \text { do } \\
\text { enfermeiro sobre a } & \text { da } \\
\text { realização } & \\
\text { classificação do risco } \\
\text { no serviço de } \\
\text { urgências }\end{array}$ & $\begin{array}{l}\text { Estudo qualitativo, } \\
\text { aplicação de entrevista } \\
\text { semiestruturada com } \\
11 \text { enfermeiros }\end{array}$ & 2014 & $\begin{array}{l}\text { SOUZA et } \\
\mathrm{al}^{14}\end{array}$ & $\begin{array}{l}\text { Invest. } \\
\text { educ. } \\
\text { enferm. } \\
\text { Medellín }\end{array}$ \\
\hline
\end{tabular}

Fonte: Do pesquisador.

Os dados foram agrupados de acordo com os aspectos que mais se destacaram e foram relevantes ao tema: Gerenciando a Equipe de Enfermagem na Sala de Emergência.

A Unidade de Urgencia e Emergencia nos coloca em diversas situações e permite o crescimento e o enriquecimento das técnicas enquanto profissional enfermeiro, sendo um dos setores de destaque profissional. Lida-se com situações tanto de urgencia, quanto de emergencia, bem como com situações de atenção primaria.

Vivencia-se uma realidade onde o Ministerio da Saude incentiva e fortalece a Atenção Básica, porém a falta de comprometimento e estruturação 
adequada dos serviços municipais leva a um costante aumento de usuários não enquadrados em situações de emergência, acentuando a demanda nas unidades de urgência e emergência ${ }^{2,7}$ e comprometendo assim a qualidade da assistência na unidade ${ }^{11}$.

Para melhor entender este contexto define-se situação de urgência como um agravo imprevisto a saúde, com ou sem risco potencial de vida, podendo progredir para um problema sério. Já em uma situação de emergência há um risco iminente de morte, necessitando o portador do agravo uma rápida intervenção ${ }^{1,2,7}$.

Para melhor estruturar a porta das unidades de urgência e emergência o Ministério da Saúde Brasileiro formulou em 2004 uma cartilha englobando a implantação do Sistema de Acolhimento com Classificação de Risco, o qual deve ser exercido por profissional enfermeiro de nível superior, onde o mesmo deve praticar uma escuta qualificada, redirecionando as mais diversas situações vivenciadas pelos usuarios ${ }^{8,11}$. Esta prática é exercida através de escalas e protocolos ${ }^{15}$.

Pesquisa de Santos et $a^{8}{ }^{8}$ levantaram os limites que permeiam o Acolhimento e a Classificação de Risco na práxis dos enfermeiros relacionam-se à falta de recursos tecnológicos e dos instrumentos necessários para a execução efetiva da consulta para classificar o risco dos usuários; dificuldade no fluxo de atendimento aos usuários devido à falta de compreensão dos mesmos mediante o protocolo.

Um fato bastante intrigante é que os profissionais vão compreender sobre Acolhimento com Classificação de Risco somente ao assumir o setor, indo ao inverso do que preconiza a Resolução, a qual diz que a Classificação de Risco é a priorização da assistência nos serviços de urgência e emergência e somente poderá ser executada pelo profissional enfermeiro ${ }^{8,15}$.

Visto que a equipe de enfermagem é o principal vínculo entre o usuário e a instituição, a atividade de acolhimento proporciona valorização e responsabilidade profissional, onde as ações e atividades de cuidado irão refletir na imagem da Instituição.

Desta forma, o enfermeiro é essencial no acolhimento e classificação de risco, sendo que o processo de trabalho é continuamente influenciado por questões 
estruturais de gestão complexas que ultrapassam o seu poder de resolução e governabilidade ${ }^{14}$.

Impulsionados pelo advento da globalização e inovações tecnológicas as instituições procuram profissionais que se destaquem uns dos outros, onde deve sobressair características empreendedoras as quais irão qualificar a assistência prestada $^{10}$.

Para a qualificação do atendimento no setor de urgência e emergência deve-se ter uma boa estruturação do atendimento pré-hospitalar que se constitui no Serviço de Atendimento Móvel de Urgência (SAMU), os serviços associados a salvamento e resgate e as Unidades de Pronto Atendimento (UPAs) onde, deve-se estabelecer um elo importante para uma reorganização dos usuários ${ }^{1,11}$.

Pesquisa de Moraes; Melleiro ${ }^{7}$ com usuários da emergência demonstrou que a dimensão que apresentou pior escore foi a estrutura, sugerindo que os usuários tendem a considerar os aspectos referentes à estrutura como os deficitários para a assistência e que a dimensão de resultado, relacionado aos efeitos da assistência na saúde do usuário obteve o melhor escore de favorabilidade.

Estudo de Amestoy et $\mathrm{al}^{10}$ destacou como limitação para o exercício profissional do enfermeiro recém-formado a escassez de recursos materiais e humanos, além da falta de experiência profissional.

Inteiramente ligado a este contexto culmina a necessidade de uma estrutura fisica adequada ao âmbito hospitalar na unidade de urgência e emergência ${ }^{7-10}$. Uma estrutura onde a equipe consiga desempenhar suas funções de forma adequada, dispondo de materiais, equipamentos e espaço físico ideal. Visto que este serviço necessita de respostas imediatas, lidando com pessoas em risco de morte, bem como podendo assegurar ao usuário um atendimento acolhedor, livre de discriminação. Visando uma relação pessoal saudável tanto para com o usuários como para a equipe profissional, onde se estabeleça igualdade no tratamento ${ }^{7}$.

No serviço hospitalar o enfermeiro ocupa uma posição central na equipe de enfermagem ${ }^{9}$. Isso não se difere em uma unidade de urgência e emergência, onde o enfermeiro é o responsavel em gerir o cuidado ao paciente, fazer previsão e recuperação de equipamentos e materiais, realizar o dimencionamento de pessoal, 
liderar a equipe e coordenar o plano assistencial ${ }^{2,11}$. Tal relevância se deve ao fato do enfermeiro trazer em sua essência a arte de cuidar.

Para gerenciar os cuidados prestados aos usuários e para liderar uma equipe de enfermagem o enfermeiro deve ter uma visão ampla da assistência e manter-se constantemente ativo com sua equipe ${ }^{9}$. Não deve apenas delegar e supervisionar as atividades, mas deve sim auxiliar na execução das mesmas, buscando o sucesso da equipe.

A comunicação efetiva entre técnicos, enfermeiros e médicos é extremamente necessária para o bom andamento do serviço ${ }^{9}$. Cada um desempenhando suas funções, porém um sendo a continuação e complementação da outra, onde cada um tem suas capacidades e dificuldades.

Pesquisa de Amestoy et al $^{10} \mathrm{com}$ enfermeiro sobre estratégias para 0 exercício da liderança na urgência e emergência destacou a importância da conversa, diálogo e do trabalho em equipe para o manejo de conflitos. Como estratégias desejadas pelos enfermeiros que poderiam ser utilizadas com a finalidade de facilitar o exercício da liderança no serviço de urgência e emergência foram citadas reuniões, capacitações e apoio psicológico para enfrentar situações desgastantes no serviço ${ }^{10}$.

Estudo de Santos et al $^{9}$ indicou como estratégias utilizadas pelos enfermeiros para promover 0 trabalho em equipe a articulação das ações profissionais; estabelecimento de relações de cooperação; construção e manutenção de vínculos amistosos e gerenciamento de conflitos.

O enfermeiro é o responsável em gerenciar os conflitos existentes na equipe caracterizando-se em uma das ações mais importantes e comumente encontradas em seu cotidiano9,12. Deve por meio de diálogo ser o mediador e negociador de situações conflitantes. Visto que conflitos são previstos, pois além de se conviver diariamente em um setor estressante, cada membro da equipe tem suas particularidades. A melhor técnica de se mediar um conflito é através da ferramenta conversa/diálogo ${ }^{10}$.

Em virtude de compromissos e atividades tão complexas e variadas, envolvendo uma gama de profissionais, o enfermeiro deve constantemente 
aprimorar seus conhecimentos, transferindo para sua equipe as inovações técnicas através de educação permanente e continuada ${ }^{8,10}$.

Quando se fala em equipe considera-se a categoria médica como parte dela, pois se todos estiverem engajados em um mesmo propósito, o de garantir melhores resultados ao paciente, o gerenciamento ira se destacar como uma prática fundamental na organização da instituição'1.

Valorizar o trabalho em equipe se faz necessário, onde deve-se fortalecer e estimular cada sujeito da equipe ${ }^{2,10}$. Com o objetivo de torná-los seres criativos e participativos no cuidado ao usuário, tendo como principal foco a saúde e o bem estar do cliente. $O$ enfermeiro precisa conquistar sua equipe tornando-se para ela um ser motivador, construindo laços de harmonia e confiança ${ }^{10}$, sendo um exemplo de postura profissional.

Para que todas estas ações sejam exercidas devemos ter planejamento, onde 0 foco principal é a organização da assistência ${ }^{2,11}$. Dinamizar a equipe de enfermagem de tal forma para que a mesma saiba se distribuir no contexto de uma situação de emergência, onde amparados pela ansiedade em uma situação de estresse consigam atuar de forma eficiente e rápida.

Identificamos que desde os primórdios da Enfermagem Moderna demonstradas por Florence Nighitingale as funções do enfermeiro são exercidas com foco gerenciador ${ }^{8,12}$. Atualmente este reconhecimento é atribuido ao enfermeiro pela Lei do Exercício Profissional e fundamentada pela atividade de Acolhimento com Classificação de Risco ${ }^{12}$.

Estudo de Santos et al ${ }^{12}$ demonstrou que a gerência é fonte de prazer quando há crescimento pessoal e profissional, reconhecimento dos colegas e satisfação do paciente; e fonte de sofrimento em função das dificuldades de relacionamento com a equipe de trabalho e da sobrecarga de trabalho do enfermeiro.

Pesquisa de Santos et $\mathrm{al}^{8}$ sobre as possibilidades que permeiam 0 Acolhimento e a Classificação de Risco na Práxis dos Enfermeiros relacionam-se com a satisfação no trabalho; gostar de trabalhar na emergência; autonomia para o acolhimento; humanização do cuidado com mudança no processo de atendimento dos pacientes mais graves; processo de educação em saúde ${ }^{8}$. 
Um dos fatores facilitadores ao papel de gerenciamento é o enfermeiro ter perfil de líder e/ou procurar desenvolver habilidades de liderança identificando-se com a profissão e exercendo suas funções com prazer, ou seja, fazer o que gosta e não fazer por fazer. Outro fator relevante é a experiência profissional, onde o profissional adquire conhecimento identificado na prática e consegue exercer atividades de gerenciamento com maior facilidade, norteado pela segurança técnica ${ }^{10}$. Pois posicionar-se frente a uma tomada de decisão e ser o líder da equipe rodeado por pessoas de maior experiência que você é muito desafiador e intrigante, conota medo e angústia.

Um fator significativo ao qual o enfermeiro identifica prazer na atividade de gerenciar é o retorno positivo, tanto da equipe profissional, quanto do paciente ${ }^{12}$. Reconhecimento este difícil de ser identificado pois entre a equipe percorre uma agitação mútua e muitas vezes frustrações pela perda do paciente e por parte do paciente a maioria das vezes ele não esta ciente de sua situação, onde seus sentidos estão rebaixados. Ficando então a critério dos familiares exercerem o sentimento de gratidão. E quando a frase "Muito obrigado" surge, nos enche de orgulho.

Gerenciar e liderar são atividades distintas visto que, para você liderar você precisa gerenciar sua equipe eficientemente, estimulando a colaboração mútua para um atendimento emergencial de qualidade ${ }^{10}$. Deve ter conhecimento pleno da rotina diária do setor, devendo estar inserido em sua realidade e ter traquejo sobre sua equipe, conduzindo-a humanamente.

O enfermeiro é o mediador das ações da equipe multiprofissional atuante no setor de urgência e emergência visando a assistência humanizada e qualificada ${ }^{9}$.

\section{CONSIDERAÇÕES FINAIS}

A funçao de enfermeiro gerenciador não se caracteriza em uma atividade atual, visto que era exercida por Florence Nighitingale desde os nossos primordios. Porem vem sofrendo muitos avanços com as inovações tecnologias, onde nos leva a mecanizar o trabalho, esquecendo o que melhor sabemos fazer, cuidar, tocar e ouvir. 
Percebe-se que o primeiro setor de trabalho do enfermeiro não deveria ser o de urgência e emergência, visto que as habilidades de líder e gerenciador devem ser aprimoradas com a vivencia prática, onde no embasamento teórico somos estimulados, porém muito pouco lapidados para a função de gerente.

O enfermeiro deve ser capacitado adequadamente desde a formação para o processo de gerenciamento em urgência e emergência frente aos conflitos no relacionamento interpessoal com a equipe e usuários; tendo conhecimento sobre 0 processo de acolhimento com classificação de risco e a práxis da humanização da assistência no serviço emergencial.

Inumeras serão as dificuldades do enfermeiro enquanto posicionado na função de gerente de equipe. Portanto deve tornar-se um ser participativo e estimulador das atividades exercidas, tanto em situações de emergencia, bem como nas atividades diarias de organização de setor, rotinas e funções.

Contudo, para colocar-se frente a esta função o profissional precisa ter uma caracteristica fundamental, a de ser fonte inspiradora para a equipe multidiciplinar, disseminando em seu meio o fazer com prazer e não o fazer por fazer.

\section{REFERENCIAS}

1. Silva DS, Bernardes A, Gabriel CS, Rocha FLR, Caldana G. A liderança do enfermeiro no contexto dos serviços de urgência e emergência. Rev. Eletr. Enf. 2014 jan/mar; 16(1):211-9.

2. Lima Neto AV, Fernandes RL, Barbosa IML, Carvalho GRP, Nunes VMA. Relacionamento interpessoal entre a equipe de uma emergência hospitalar: um estudo qualitativo sob o olhar de enfermeiros. Rev. Enf. 2015 jan/abr; 18(1):75-87.

3. Mendes KDS, Silveira RCCP, Galvão CM. Revisão integrativa: método de pesquisa para a incorporação de evidências na saúde e na Enfermagem. Texto contexto - enferm. $2008 \mathrm{dez}$; 17(4): 758-764

4. Souza MT; Silva MD; Carvalho R. Revisão integrativa: o que é e como fazer. Einstein 2010 mar; 8(1):102-106. 
5. Botelho LLR; Cunha CCA; Macedo M. O Método da Revisão Integrativa nos Estudos Organizacionais. Gestão e Sociedade 2011 dez; 5(11): 121-136.

6. Crossetti MGO. Revisão integrativa de pesquisa na Enfermagem: o rigor científico que Ihe é exigido. Rev.Gaucha Enferm. 2012 jun; 33(2):8-9.

7. Morais AS, Melleiro MM. A qualidade da assistência de enfermagem em uma unidade de emergência: a percepção do usuário. Rev. Eletr. Enf. 2013 jan/mar; 15(1):112-20.

8. Araujo YB, Ferreira LBA, Santos CM, Silva TMF, Gomes TSM. Acolhimento e classificação de risco nos serviços de urgência e emergência: limites e possibilidades uma questão para os enfermeiros. Persp. On line: biol. Saúde, Campos de Goytacazes 2014; 15(4):25-49.

9. Santos JLG, Lima MADS, Pestana AL, Colomé ICS, Erdmann AL. Estratégias utilizadas pelos enfermeiros para promover o trabalho em equipe em um serviço de emergência. Rev. Gaúcha Enferm. 2016; 37(1): e50178.

10. Amestoy SC, Lopes RF, Santos BP, Dornelles C, Fuculo Junior PRB, Santos EA. Exercício da liderança do enfermeiro em um serviço de urgência e emergência. Rev. Elet. Gestão e Saúde 2016; 1:38-51.

11. Zambiazi BRB, Costa AM. Gerenciamento de enfermagem em unidade de emergência: dificuldades e desafios. RAS 2013 out/dez; 15(61):169-176.

12. Santos JLG, Prochnow AG, Silva DC, Silva RM, Leite JL, Erdmann AL. Prazer e sofrimento no exercício gerencial do enfermeiro no contexto hospitalar. Esc. Anna Nery 2013 mar; 17(1): 97-103.

13. Carvalho GPA. Sistematização da assistência de enfermagem e o gerenciamento do cuidado: revisão de literatura. Sínteses: Rev. Elet. SIMTEC 2016 out $27 ; 0(6)$ : 266-266. 
14. Souza CG, Diniz AS, Silva LLT, Mata LRF, Chianca TCM. Percepção do enfermeiro sobre a realização da classificação do risco no serviço de urgências. Invest. educ. enferm. Medellín 2014 jan/abr; 32(1):78-86.

15. Brasil. Ministério da Saúde. Secretaria de Atenção à Saúde. Departamento de Atenção Especializada. Manual instrutivo da Rede de Atenção às Urgências e Emergências no Sistema Único de Saúde (SUS). Brasília: Ministério da Saúde, 2013. $84 \mathrm{p}$. 